Palavras chave: Produção

Incrementos

Modelos biológicos

Análise de tronco

Anéis de crescimento

Histórico:

Recebido 06/05/201 I

Aceito $21 / 01 / 2015$

Keywords: Yield

Increments

Biological models

Complete stem analysis

Growth rings

Correspondência: samachado@ufpr.br

DOI:
Sebastião do Amaral Machado', Ronan Felipe de Souza', Luiza Maria Teóphilo Aparecido², Andressa Ribeiro ${ }^{3}$, Bruno Henrique Czelusniak'

\section{EVOLUÇÃO DAS VARIÁVEIS DENDROMÉTRICAS DA BRACATINGA POR CLASSE DE SÍTIO}

RESUMO: A identificação do ritmo de crescimento das variáveis dendrométricas de espécies florestais com o uso da análise de tronco é rápida, eficiente e de grande importância na tomada de decisões para o manejador florestal. Sendo assim, objetivouse neste trabalho analisar a evolução das curvas de produção, Incremento Médio Anual (IMA) e Incremento Corrente Anual (ICA) da altura, diâmetro, área transversal e volume individual da Bracatinga (Mimosa scabrella), em diferentes classes de sítio, bem como, ajustar modelos matemáticos para expressar a evolução dessas variáveis, ao longo dos anos. A base de dados proveio da análise completa de tronco de 29 árvores, coletadas na região metropolitana de Curitiba, com idades variando de sete a 18 anos, distribuídas em três classes de sítio. Após as medições e processamento básico dos dados, ajustaram-se seis modelos matemáticos conhecidos na literatura, para cada uma das quatro variáveis dendrométricas em análise, independentemente para cada sítio. $O$ melhor modelo foi escolhido com base no coeficiente de determinação ajustado $\left(R_{\mathrm{aj}}^{2}\right)$, erro padrão de estimativa em percentagem $\left(\mathrm{S}_{\mathrm{yx}} \%\right)$ e análise gráfica de resíduos. Os resultados mostraram que o modelo de Chapman-Richards foi o de melhor ajuste para quase todas as variáveis em análise dentro dos três sítios. Verificou-se, também, que a ordem de culminação do incremento corrente anual seguiu a ordem cronológica esperada, isto é, primeiro a altura, seguida pelo diâmetro, área transversal e, por último, o volume, em todos os sítios.

\section{EVOLUTION OF DENDROMETRIC VARIABLES OF Mimosa scabrella BY SITE CLASS}

ABSTRACT: The identification of the growth rhythm of the dendrometric variables of forest species with the use of stem analysis is quick, efficient and of great importance in the decision making by forest managers. Thus, the objectives of this research were to analyze the annual evolution of the yield curves, Mean Annual Increment (MAI) and Current Annual Increment (CAI) in height, diameter, cross sectional area and volume of individual Mimosa scabrella Benth (Bracatinga) in different site classes, and also, to fit mathematical models to express the evolution of these variables over the years. The database came from complete stem analysis of 29 Mimosa scabrella trees collected in the metropolitan region of Curitiba, with ages ranging from seven to 18 years, distributed in three site classes. After the measurements and basic data processing, six mathematical models were adjusted for each one of the four dendrometric variables, independently for each site. The best model was chosen based on the adjusted coefficient of determination $\left(\mathrm{R}^{2} \mathrm{aj}\right)$, relative standard error of estimate $\left(\mathrm{S}_{\mathrm{yx}} \%\right)$ and residual graphic analysis. The results showed that the Chapman-Richards model presented the best fitting for almost all analyzed variables in all three sites. It was also verified that the culmination order of the current annual increment followed the expected chronological order, that is, first height, then diameter, cross sectional area, and finally, volume, in all the sites.

' Universidade Federal do Paraná - Curitiba, Paraná, Brasil

2 Universidade Texas A\&M - College Station, Texas, Estados Unidos

${ }^{3}$ Universidade Federal do Piauí - Bom Jesus, Piauí, Brasil 


\section{INTRODUÇÃO}

A identificação do ritmo de crescimento das variáveis dendrométricas de espécies florestais é de grande importância para a tomada de decisões pelo manejador florestal. Assmann (1970) destacou que o conhecimento do comportamento de cada variável dendrométrica permite a identificações das relações entre a produção, incremento corrente anual (ICA) e incremento médio anual (IMA).

Quando uma espécie apresenta anéis de crescimento visíveis, como éo caso da Bracatinga, a técnica de análise de tronco permite recompor o crescimento passado das árvores de maneira relativamente rápida e simples. Com base na análise de tronco, pode-se avaliar a produtividade de um sítio, o crescimento e qualidade de determinado indivíduo em diferentes períodos de sua vida (FINGER, 1992).

Modelos biológicos de crescimento têm sido amplamente utilizados para descrever o crescimento de variáveis dendrométricas de árvores e povoamentos florestais, como pode ser observado nos trabalhos de Ferraz Filho et al. (20II), Scolforo e Machado (1988) e Tonini et al. (200I). Tais modelos são amplamente empregados por permitirem a projeção da variável de interesse até idades avançadas, superiores ao limite dos dados observados. Tonini et al. (2008) com o objetivo de estudar o crescimento de espécies nativas plantadas na Amazônia, ajustaram o modelo de ChapmanRichards para analisar a evolução do diâmetro e da altura, obtendo resultados satisfatórios para ajustes matemáticos e garantindo uma aferição clara nos resultados do experimento.

A Bracatinga (Mimosa scabrella Benth) é característica e exclusiva das regiões das matas de pinhais, principalmente de associações secundárias, onde, frequentemente, chega a formar povoamentos puros. É uma espécie nativa e, há mais de 100 anos, tem seu uso norteado pelo mercado de lenha para queima direta.

Atualmente, a demanda por madeira da espécie está voltada para atender parte das necessidades relativas de madeira para energia, assim como para outras finalidades industriais, tais como: serraria, laminação, movelaria e placas e painéis (SILVA et al., 2006). Nesse sentido, esforços vêm sendo realizados nos últimos anos em prol do desenvolvimento nas técnicas de manejo da espécie na Região Metropolitana de Curitiba (RMC) e, com a publicação de uma classificação de sítios definitiva para a região em 20I I (MACHADO et al., 20 I I), tendo em vista que o conhecimento do comportamento das variáveis dendrométricas por sítio se faz necessário, buscando subsidiar o aprimoramento das técnicas de manejo já empregadas para essa espécie.

Nesse contexto, objetivou-se neste trabalho, identificar o comportamento das curvas de produção, IMA e ICA da altura, diâmetro, área transversal e volume individual da Bracatinga em diferentes classes de sítio, bem como, ajustar modelos matemáticos para expressar a evolução dessas variáveis ao longo dos anos.

\section{MATERIAL E MÉTODOS}

\section{Caracterização da área de estudo}

As árvores utilizadas para este trabalho foram coletadas na região norte de Curitiba, conhecida como Vale do Ribeira. O clima predominante na região, segundo a classificação de Köppen, é do tipo Cfb, com precipitação em todas as estações; a temperatura média do mês mais quente é inferior a $22^{\circ} \mathrm{C}$. A geologia da região é de origem metamórfica, resultando em uma topografia fortemente ondulada com solos muito heterogêneos no que se refere à profundidade $\mathrm{e}$ fertilidade. Os solos desta região, na sua maioria, são classificados como Cambissolo e apresentam-se pobres, pouco desenvolvidos, ácidos, com teores elevados de alumínio e baixa saturação de bases (CARPANEZZI et al., 1988).

\section{Base de dados}

A base de dados utilizada proveio da análise de tronco completa de 29 árvores dominantes escolhidas de acordo com o critério de Assmann, distribuídas nas três classes de sítio existentes para a região metropolitana de Curitiba. Tais indivíduos foram coletados em parcelas existentes em bracatingais da região, onde as alturas dominantes foram determinadas previamente, procurando-se cobrir igualmente as três classes de sítio. Isso foi possível para as idades mais jovens e sítios mais produtivos. No entanto, a raridade de se encontrar povoamentos com idades acima de 14 anos e com baixa produtividade, resultou em baixo número de árvores amostradas com idades superiores a 14 anos, notadamente para o sítio III, como pode ser observado na Tabela I.

Após a derrubada das árvores, foram retirados discos nas alturas de 0,10; 0,70 e I,30 metros a partir do solo e, a partir daí, a cada dois metros até o ponto de inversão morfológica. Desse ponto em diante foi coletado um disco a cada metro ao longo do galho classificado como principal. 
TABELA 1 Frequência amostral por classe de idade e classe de sítio.

TABLE 1 Sampling frequency by age and site class.

\begin{tabular}{cccc}
\hline Idade & Sítio I & Sítio II & Sítio III \\
\hline 1 & 11 & 12 & 7 \\
2 & 11 & 12 & 7 \\
3 & 11 & 12 & 7 \\
4 & 11 & 12 & 7 \\
5 & 11 & 12 & 7 \\
6 & 11 & 12 & 7 \\
7 & 11 & 12 & 7 \\
8 & 11 & 12 & 6 \\
9 & 11 & 12 & 6 \\
10 & 9 & 11 & 5 \\
11 & 9 & 11 & 5 \\
12 & 8 & 9 & 5 \\
13 & 8 & 8 & 5 \\
14 & 6 & 5 & 4 \\
15 & 4 & 3 & 2 \\
16 & 2 & 3 & 1 \\
17 & 1 & 3 & 1 \\
18 & 1 & 1 &
\end{tabular}

\section{Análise de tronco completa}

Os discos foram trazidos do campo em sacos apropriados e permaneceram em local arejado por alguns dias, objetivando a perda de umidade, sendo, então, colocados para secar em estufa, à temperatura de $28^{\circ} \mathrm{C}$ por dois dias. Em seguida, foi efetuado o lixamento de uma das superfícies de cada disco, facilitando, assim, o processo de identificação e medição dos anéis de crescimento.

A medição dos discos foi realizada após traçados quatro raios sobre a superfície do disco lixada e que foram definidos a partir de $45^{\circ}$ do maior raio existente em cada fatia, seguindo a metodologia definida por Barusso (1977). A marcação, contagem e medição dos raios dos anéis foram feitas com uma régua comum, com aproximação em milímetros, partindo-se da medula até a borda do disco.

\section{Processamento dos dados}

O diâmetro dos anéis de crescimento foi calculado a partir dos raios medidos ao longo dos anos nas diferentes alturas. A altura de cada anel foi determinada do topo para a base, usando o método do paralelismo ou proporcionalidade entre alturas de anéis subsequentes, como descrito por Machado et al. (2010). Os volumes foram calculados através da fórmula de Smalian, associada à fórmula do cone para a secção da ponta. A fim de montar a matriz final dos dados processados, fez-se a classificação das árvores por classe de sítio de acordo com Machado et al. (20II) para, então, calcular a média de cada variável por idade dentro de cada uma das classes de sítio.

\section{Modelagem da produção}

Utilizando a média aritmética dos dados agrupados de cada variável por classe de sítio, realizou-se a modelagem da produção ao longo da vida das árvores em cada sítio. Os modelos testados, independentemente para cada sítio e para cada uma das quatro variáveis são apresentados na Tabela 2.

TABELA 2 Modelos testados. TABLE 2 Tested models.

\begin{tabular}{lc}
\hline Autor & Modelo \\
\hline Chapman-Richards & $\mathrm{Y}=\mathrm{b}_{0}\left(1-\mathrm{e}^{-\mathrm{b}_{1} \mathrm{t}}\right)^{\mathrm{b}_{2}}$ \\
Bertalanffy & $\mathrm{Y}=\mathrm{b}_{0}\left(1-\mathrm{e}^{-\mathrm{b}_{1} \mathrm{t}}\right)^{3}$ \\
Mitscherlich & $\mathrm{Y}=\mathrm{b}_{0}\left(1-\mathrm{e}^{-\mathrm{b}_{1} \mathrm{t}}\right)$ \\
Gompertz & $\mathrm{Y}=\mathrm{b}_{0}\left(\mathrm{e}^{\left.-\mathrm{b}_{1} \mathrm{t}\right)^{-\mathrm{b}_{2} \mathrm{t}}}\right.$ \\
Logístico & $\mathrm{Y}=\frac{\mathrm{b}_{0}}{\left(1+\mathrm{b}_{1} \mathrm{e}^{-\mathrm{b}_{2} \mathrm{t}}\right)}$ \\
Backman Modificado & $\mathrm{Y}=\mathrm{b}_{0}+\left(\mathrm{b}_{1} \mathrm{t}\right)+\left(\mathrm{b}_{2} \mathrm{t}^{2}\right)$ \\
\hline
\end{tabular}

Sendo: $\mathrm{Y}=$ Variável de interesse; $\mathrm{t}=$ Idade em anos e $b_{0}, b_{1}$ e $b_{2}=$ coeficientes a serem estimados.

No total foram realizados 72 ajustes, onde cada modelo foi ajustado 12 vezes (quatro variáveis em três sítios). $\bigcirc$ grande número de ajustes tornou necessária a utilização de um ranking entre os seis modelos para facilitar a identificação do melhor. Para definir a pontuação no ranking, foram considerados o coeficiente de determinação ajustado $\left(R^{2}\right)$ e $\circ$ erro padrão da estimativa percentual $\left(\mathrm{S}_{\mathrm{yx}} \%\right)$. Foi considerado mais acurado o modelo que apresentou maior valor de $\mathrm{R}^{2} \mathrm{e}$ menor valor de $\mathrm{S}_{y x} \%$, atribuindo-se ao mesmo valor igual a um. Para o modelo com $\mathrm{R}^{2}$ mais baixo e $\mathrm{S}_{\mathrm{yx}} \%$ mais alto foi atribuído valor igual a seis.

A somatória da pontuação de cada modelo resultou em uma classificação geral, onde o modelo com menor pontuação foi selecionado para descrever o comportamento das quatro variáveis dendrométricas nos diferentes sítios. Com base nos valores ajustados do modelo selecionado, calcularam-se os incrementos 
médios anuais (IMA) e incremento corrente anual (ICA), bem como a produção para as quatro variáveis nos diferentes sítios.

\section{RESULTADOS E DISCUSSÃO}

método de análise de tronco utilizado foi eficiente para obter o diâmetro e altura das árvores nas diferentes idades. A existência de uma sólida base de dados permitiu a aplicação da metodologia utilizada para se fazer o estudo das relações de produção, ICA e IMA da espécie nas diferentes classes de sítio da região.

A base final de dados foi composta por 135 pontos no sítio I, I 39 pontos no sítio II e 67 pontos no sítio III, totalizando $34 \mathrm{I}$ pontos amostrais. Cada ponto correspondeu a um valor de altura $(\mathrm{m})$, diâmetro $(\mathrm{cm})$, área transversal $\left(\mathrm{cm}^{2}\right)$ e volume $\left(\mathrm{dm}^{3}\right)$.

\section{Modelagem das variáveis dendrométricas}

Todos os modelos testados apresentaram distribuição de resíduos homogênea, independente e pouco dispersa ao longo da linha zero. O modelo de Mitscherlich foi o modelo que apresentou as maiores dispersões de resíduos, com destaque para área transversal e volume.

O ordenamento (ranking) dos modelos ajustados foi baseado nas estatísticas $\mathrm{R}^{2}$ e $\mathrm{S}_{\mathrm{yx}} \%$. Ao somar as pontuações alcançadas por cada modelo, verificou-se que $o$ de Chapman-Richards atingiu a menor soma (20 pontos), sendo o primeiro em $50 \%$ dos casos e sempre se classificando em segundo ou terceiro lugar para as outras situações analisadas. $O$ segundo melhor modelo foi o de Gompertz com 35 pontos, seguido pelo modelo de Backman Modificado com 36 pontos. O pior de todos os modelos testados foi o modelo Logístico com 59 pontos.

$\mathrm{Na}$ Tabela 3, são apresentados os coeficientes ajustados e as estatísticas para o modelo de ChapmanRichards. Os valores do coeficiente $b_{0}$ correspondem à assíntota da produtividade de cada variável.

Tonini et al. (2009), realizando estudo de crescimento de Teca, utilizaram o modelo de ChapmanRichards para descrever o crescimento em altura, diâmetro e volume. Para todas as variáveis, o modelo se portou de forma satisfatória, permitindo aos autores analisar as curvas de incremento das mesmas. Para Hess e Schneider (2010) o modelo de Chapman-Richards não apresentou os mesmos resultados. Apesar de bons ajustes observados para esse modelo, os autores selecionaram o modelo de Backman como o mais
TABELA 3 Coeficientes e estatísticas do ajuste do modelo de Chapman-Richards.

TABLE 3 Coefficients and statistics for the Chapman-Richards model.

\begin{tabular}{cccccc}
\hline $\begin{array}{c}\text { Sítio e estatísticas } \\
\text { do ajuste }\end{array}$ & $\begin{array}{c}\text { Altura } \\
(\mathrm{m})\end{array}$ & $\begin{array}{c}\text { Diâmetro } \\
(\mathrm{cm})\end{array}$ & $\begin{array}{c}\text { Área } \\
\text { transversal } \\
\left(\mathrm{cm}^{2}\right)\end{array}$ & $\begin{array}{c}\text { Volume } \\
\left(\mathrm{dm}^{3}\right)\end{array}$ \\
\hline & $\mathrm{b}_{0}$ & 20,8007 & 22,1981 & 445,4006 & 548,5202 \\
& $\mathrm{~b}_{1}$ & 0,2408 & 0,1506 & 0,1223 & 0,1106 \\
& $\mathrm{~b}_{2}$ & 1,2007 & 1,2942 & 2,2632 & 2,6560 \\
& $\mathrm{R}^{2}$ & 0,9969 & 0,9960 & 0,9960 & 0,9962 \\
& $\mathrm{~S}_{\mathrm{yx}} \%$ & 2,23 & 3,28 & 4,90 & 6,73 \\
\hline & $\mathrm{b}_{0}$ & 16,9600 & 19,1671 & 330,5250 & 515,4079 \\
& $\mathrm{~b}_{1}$ & 0,2185 & 0,1587 & 0,1336 & 0,0881 \\
& $\mathrm{~b}_{2}$ & 1,2870 & 1,6290 & 2,8363 & 3,0529 \\
& $\mathrm{R}^{2}$ & 0,9991 & 0,9988 & 0,9994 & 0,9998 \\
& $\mathrm{~S}_{\mathrm{y} \%} \%$ & 1,29 & 2,07 & 2,00 & 1,54 \\
\hline & $\mathrm{b}_{0}$ & 14,6535 & 13,4943 & 178,1603 & 142,0269 \\
& $\mathrm{~b}_{1}$ & 0,1971 & 0,2065 & 0,1469 & 0,1456 \\
& $\mathrm{~b}_{2}$ & 1,2938 & 1,8505 & 2,7624 & 3,5982 \\
& $\mathrm{R}^{2}$ & 0,9903 & 0,9983 & 0,9984 & 0,9990 \\
& $\mathrm{~S}_{\mathrm{yx}} \%$ & 4,46 & 2,37 & 3,26 & 3,02 \\
\hline
\end{tabular}

acurado para descrever o crescimento em volume de Araucaria angustifolia.

Sanquetta et al. (2010), ao ajustarem curvas de crescimento para a espécie Nectandra grandiflora por meio de análise de tronco, observaram que dentre os seis modelos testados, os de Gompertz e Logístico resultaram em um ajuste insatisfatório. Os modelos de ChapmanRichards, Korf e Weibull resultaram em ajustes satisfatórios e, entre eles, Weibull foi considerado o melhor.

\section{Evolução e análise das variáveis dendrométricas por classe de sítio}

O estudo da evolução das variáveis dendrométricas foi realizado com base nas curvas de produção, ICA e IMA, provenientes do ajuste do modelo de Chapman-Richards. Assim, para cada classe de sítio foi construída uma tabela com os valores anuais de produção de cada variável, bem como, gerados os respectivos gráficos; por limitação de espaço, no entanto, são apresentadas apenas as curvas para o sítio II (Figura I).

Nos três sítios analisados, verificou-se a seguinte sequência cronológica de assíntota no ICA das variáveis dendrométricas: altura, diâmetro, área transversal e volume. Esse resultado está de acordo com a hipótese tradicionalmente aceita em estudos de crescimento e produção florestal. No 
que se refere à intersecção das curvas de ICA e IMA, observou-se, também, a mesma sequência para as variáveis estudadas. Os valores destacados na Tabela 4 correspondem ao ICA máximo encontrado para a variável e a intersecção das curvas de ICA e IMA no sítio I.
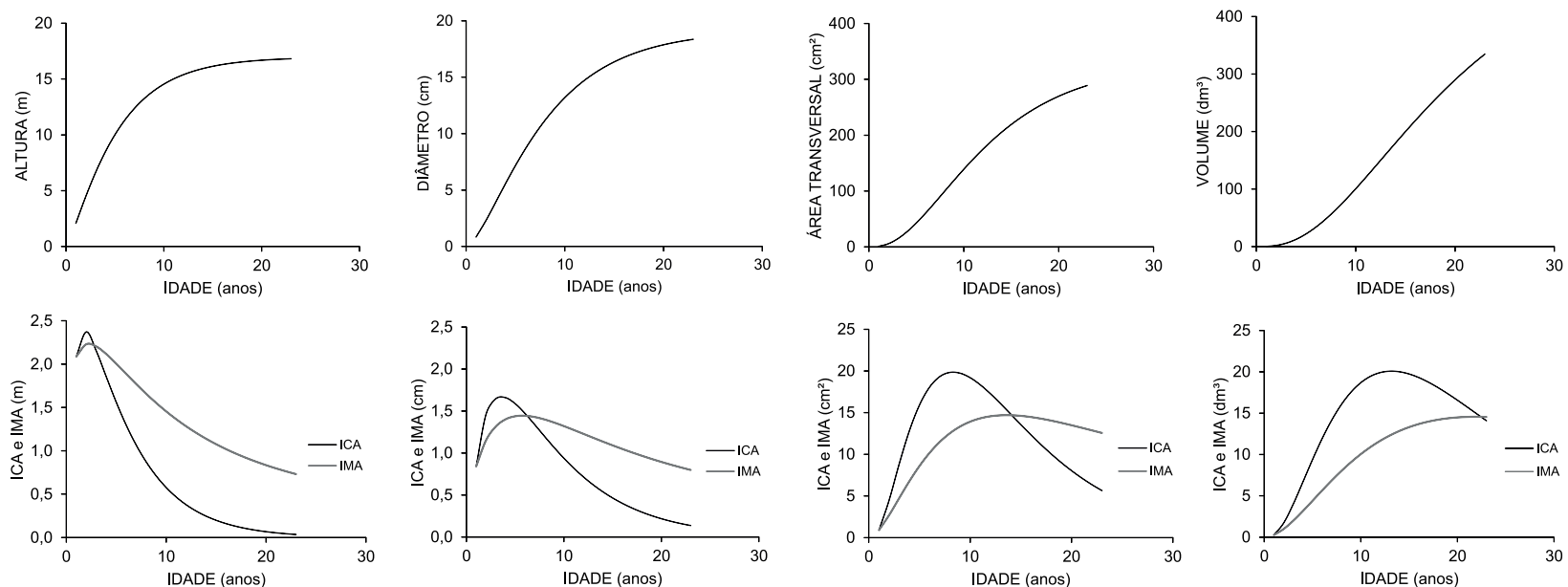

FIGURA 1 Representação gráfica das curvas de produção, ICA e IMA para as quatro variáveis analisadas no sítio II.

FIGURE 1 Graphic representation of yield, CAI and MAI curves for the four analyzed variables on site II.

TABELA 4 Produção, ICA e IMA para as variáveis analisadas no sítio I.

TABLE 4 Yield, CAI and MAI for the analyzed variables on site I.

\begin{tabular}{|c|c|c|c|c|c|c|c|c|c|c|c|c|}
\hline Idade & $\mathrm{h}$ & ICA h & IMA h & dap & ICA dap & IMA dap & $\mathrm{g}$ & ICA g & IMAg & v & ICA v & IMA v \\
\hline 1 & 3,27 & 3,27 & 3,27 & 1,74 & 1,74 & 1,74 & 3,34 & 3,34 & 3,34 & 1,37 & 1,37 & 1,37 \\
\hline 2 & 6,55 & 3,29 & 3,28 & 3,88 & 2,14 & 1,94 & 14,03 & 10,69 & 7,02 & 7,47 & 6,10 & 3,74 \\
\hline 3 & 9,36 & 2,81 & 3,12 & 5,99 & 2,11 & 2,00 & 30,81 & 16,77 & 10,27 & 19,07 & 11,60 & 6,36 \\
\hline 4 & 11,68 & 2,31 & 2,92 & 7,96 & 1,96 & 1,99 & 51,95 & 21,14 & 12,99 & 35,68 & 16,62 & 8,92 \\
\hline 5 & 13,55 & 1,88 & 2,71 & 9,74 & 1,78 & 1,95 & 75,91 & 23,96 & 15,18 & 56,41 & 20,73 & 11,28 \\
\hline 6 & 15,06 & 1,51 & 2,51 & 11,33 & 1,60 & 1,89 & 101,41 & 25,50 & 16,90 & 80,22 & 23,82 & 13,37 \\
\hline 7 & 16,26 & 1,20 & 2,32 & 12,75 & 1,42 & 1,82 & 127,45 & 26,04 & 18,21 & 106,15 & 25,93 & 15,16 \\
\hline 8 & 17,22 & 0,96 & 2,15 & 14,00 & 1,25 & 1,75 & 153,30 & 25,85 & 19,16 & 133,32 & 27,17 & 16,66 \\
\hline 9 & 17,97 & 0,76 & 2,00 & 15,09 & 1,09 & 1,68 & 178,41 & 25,11 & 19,82 & 161,00 & 27,68 & 17,89 \\
\hline 10 & 18,57 & 0,60 & 1,86 & 16,05 & 0,96 & 1,60 & 202,42 & 24,01 & 20,24 & 188,60 & 27,60 & 18,86 \\
\hline 11 & 19,05 & 0,47 & 1,73 & 16,88 & 0,83 & 1,53 & 225,08 & 22,66 & 20,46 & 215,67 & 27,07 & 19,61 \\
\hline 12 & 19,42 & 0,37 & 1,62 & 17,60 & 0,72 & 1,47 & 246,26 & 21,18 & 20,52 & 241,85 & 26,18 & 20,15 \\
\hline 13 & 19,71 & 0,29 & 1,52 & 18,23 & 0,63 & 1,40 & 265,89 & 19,63 & 20,45 & 266,90 & 25,05 & 20,53 \\
\hline 14 & 19,95 & 0,23 & 1,42 & 18,77 & 0,54 & 1,34 & 283,96 & 18,07 & 20,28 & 290,65 & 23,75 & 20,76 \\
\hline 15 & 20,13 & 0,18 & 1,34 & 19,24 & 0,47 & 1,28 & 300,50 & 16,55 & 20,03 & 312,99 & 22,34 & 20,87 \\
\hline 16 & 20,27 & 0,14 & 1,27 & 19,65 & 0,41 & 1,23 & 315,58 & 15,08 & 19,72 & 333,88 & 20,89 & 20,87 \\
\hline 17 & 20,38 & 0,11 & 1,20 & 20,00 & 0,35 & 1,18 & 329,27 & 13,69 & 19,37 & 353,30 & 19,42 & 20,78 \\
\hline 18 & 20,47 & 0,09 & 1,14 & 20,31 & 0,30 & 1,13 & 341,65 & 12,38 & 18,98 & 371,27 & 17,97 & 20,63 \\
\hline 19 & 20,54 & 0,07 & 1,08 & 20,57 & 0,26 & 1,08 & 352,82 & 11,17 & 18,57 & 387,83 & 16,56 & 20,41 \\
\hline 20 & 20,60 & 0,05 & 1,03 & 20,80 & 0,23 & 1,04 & 362,87 & 10,05 & 18,14 & 403,05 & 15,21 & 20,15 \\
\hline 21 & 20,64 & 0,04 & 0,98 & 20,99 & 0,19 & 1,00 & 371,89 & 9,02 & 17,71 & 416,98 & 13,93 & 19,86 \\
\hline 22 & 20,68 & 0,03 & 0,94 & 21,16 & 0,17 & 0,96 & 379,98 & 8,09 & 17,27 & 429,71 & 12,73 & 19,53 \\
\hline 23 & 20,70 & 0,03 & 0,90 & 21,30 & 0,14 & 0,93 & 387,22 & 7,24 & 16,84 & 441,30 & 11,60 & 19,19 \\
\hline
\end{tabular}

Sendo: $\mathrm{h}=$ Altura $(\mathrm{m}) ;$ dap $=$ Diâmetro à $1,30 \mathrm{~cm}$ do solo $(\mathrm{cm}) ; \mathrm{g}=$ Área transversal $\left(\mathrm{cm}^{2}\right) ; \mathrm{v}=$ volume individual $\left(\mathrm{dm}^{3}\right)$. 
da ordem de 7,4 cm, portanto, próximos aos valores relatados neste trabalho para as idades de 5 e 6 anos, onde o diâmetro variou de 7,19 a 8,66, respectivamente.

No que se refere à altura, Carpanezzi et al. (1988), se valendo de valores de altura média aritmética dos povoamentos, encontraram valores de 10,73 $\mathrm{m}$ e I3,6 m para idades respectivas de 4,5 e 12 anos. Valores próximos foram também encontrados para o sítio II aos 5 e 12 anos (Tabela 5).

Para o sítio III, a mesma sequência cronológica da culminação do ICA foi verificada, como pode ser observado na Tabela 6. A altura teve valor assintótico da produção $\left(b_{0}\right)$ igual à $14,65 \mathrm{~m}$, o diâmetro apresentou assíntota de produção de $13,49 \mathrm{~cm}$. A área transversal e o volume apresentaram assíntota de produção igual à $178,16 \mathrm{~cm}^{2}$ e $142,03 \mathrm{dm}^{3}$, respectivamente.

Teoricamente, espera-se que as variáveis dendrométricas do povoamento nos melhores sítios apresentem valores superiores de produção, bem como, ocorra intersecção das curvas de incremento, antecipadamente, em relação aos demais. Na presente pesquisa, essa teoria foi confirmada para todas as variáveis.
O efeito do sítio sobre a variável altura foi verificado, uma vez que os valores de produção foram maiores para o sítio I, seguido pelo sítio II e então pelo sítio III. Machado et al. (200I), avaliando o efeito do sítio na evolução das variáveis destacaram, por meio de análise de variância, que o sítio exerce efeito significativo sobre a altura média e altura dominante para indivíduos de bracatinga. O máximo ICA para os três sítios ocorreu na mesma idade (dois anos). A intersecção das curvas ICA e IMA nos sítios I e II ocorreu também na mesma idade (três anos) e para o sítio III este ponto foi observado aos quatro anos.

A produção em diâmetro no sítio I foi superior à do sítio II e que, por sua vez, foi superior ao sítio III. Nutto (200l) também encontrou em seu trabalho um resultado similar no que se refere ao efeito do sítio sobre o crescimento em diâmetro de Araucaria angustifolia. A culminação do ICA para o sítio I ocorreu aos dois anos e, para os demais sítios ocorreu aos quatro anos. A intersecção das curvas de ICA e IMA ocorreu antecipadamente também para o sítio I, aos quatro anos, enquanto para os demais ocorreu aos sete anos.

TABELA 5 Produção, ICA e IMA para as variáveis analisadas no sítio II.

TABLE 5 Yield, CAI and MAI for the analyzed variables on site II.

\begin{tabular}{|c|c|c|c|c|c|c|c|c|c|c|c|c|}
\hline Idade & $\mathrm{h}$ & ICA h & IMA h & dap & ICA dap & IMA dap & $\mathrm{g}$ & ICA g & IMA g & v & ICA v & IMA v \\
\hline 1 & 2,09 & 2,09 & 2,09 & 0,84 & 0,84 & 0,84 & 0,91 & 0,91 & 0,91 & 0,27 & 0,27 & 0,27 \\
\hline 2 & 4,46 & 2,37 & 2,23 & 2,30 & 1,46 & 1,15 & 5,41 & 4,50 & 2,70 & 1,97 & 1,70 & 0,99 \\
\hline 3 & 6,61 & 2,15 & 2,20 & 3,94 & 1,64 & 1,31 & 14,28 & 8,87 & 4,76 & 5,97 & 4,00 & 1,99 \\
\hline 4 & 8,46 & 1,85 & 2,12 & 5,60 & 1,66 & 1,40 & 27,11 & 12,83 & 6,78 & 12,65 & 6,68 & 3,16 \\
\hline 5 & 10,02 & 1,56 & 2,00 & 7,19 & 1,59 & 1,44 & 43,04 & 15,93 & 8,61 & 22,04 & 9,40 & 4,41 \\
\hline 6 & 11,32 & 1,30 & 1,89 & 8,66 & 1,47 & 1,44 & 61,11 & 18,07 & 10,18 & 33,99 & 11,95 & 5,67 \\
\hline 7 & 12,39 & 1,07 & 1,77 & 10,00 & 1,34 & 1,43 & 80,44 & 19,33 & 11,49 & 48,18 & 14,19 & 6,88 \\
\hline 8 & 13,26 & 0,87 & 1,66 & 11,20 & 1,20 & 1,40 & 100,26 & 19,83 & 12,53 & 64,26 & 16,08 & 8,03 \\
\hline 9 & 13,97 & 0,71 & 1,55 & 12,27 & 1,06 & 1,36 & 120,00 & 19,74 & 13,33 & 81,84 & 17,58 & 9,09 \\
\hline 10 & 14,55 & 0,58 & 1,45 & 13,20 & 0,94 & 1,32 & 139,19 & 19,19 & 13,92 & 100,54 & 18,70 & 10,05 \\
\hline 11 & 15,01 & 0,47 & 1,36 & 14,02 & 0,82 & 1,27 & 157,53 & 18,34 & 14,32 & 120,00 & 19,46 & 10,91 \\
\hline 12 & 15,39 & 0,38 & 1,28 & 14,74 & 0,72 & 1,23 & 174,80 & 17,27 & 14,57 & 139,91 & 19,91 & 11,66 \\
\hline 13 & 15,70 & 0,31 & 1,21 & 15,36 & 0,62 & 1,18 & 190,87 & 16,07 & 14,68 & 160,00 & 20,08 & 12,31 \\
\hline 14 & 15,94 & 0,25 & 1,14 & 15,90 & 0,54 & 1,14 & 205,69 & 14,82 & 14,69 & 180,01 & 20,01 & 12,86 \\
\hline 15 & 16,14 & 0,20 & 1,08 & 16,36 & 0,46 & 1,09 & 219,25 & 13,56 & 14,62 & 199,75 & 19,74 & 13,32 \\
\hline 16 & 16,30 & 0,16 & 1,02 & 16,76 & 0,40 & 1,05 & 231,58 & 12,33 & 14,47 & 219,06 & 19,31 & 13,69 \\
\hline 17 & 16,43 & 0,13 & 0,97 & 17,11 & 0,34 & 1,01 & 242,73 & 11,15 & 14,28 & 237,81 & 18,75 & 13,99 \\
\hline 18 & 16,53 & 0,10 & 0,92 & 17,41 & 0,30 & 0,97 & 252,77 & 10,03 & 14,04 & 255,90 & 18,09 & 14,22 \\
\hline 19 & 16,62 & 0,08 & 0,87 & 17,66 & 0,25 & 0,93 & 261,76 & 9,00 & 13,78 & 273,26 & 17,36 & 14,38 \\
\hline 20 & 16,68 & 0,07 & 0,83 & 17,88 & 0,22 & 0,89 & 269,80 & 8,04 & 13,49 & 289,83 & 16,57 & 14,49 \\
\hline 21 & 16,74 & 0,05 & 0,80 & 18,07 & 0,19 & 0,86 & 276,96 & 7,16 & 13,19 & 305,59 & 15,76 & 14,55 \\
\hline 22 & 16,78 & 0,04 & 0,76 & 18,23 & 0,16 & 0,83 & 283,33 & 6,37 & 12,88 & 320,51 & 14,92 & 14,57 \\
\hline 23 & 16,82 & 0,03 & 0,73 & 18,36 & 0,14 & 0,80 & 288,98 & 5,65 & 12,56 & 334,60 & 14,09 & 14,55 \\
\hline
\end{tabular}

Sendo: $\mathrm{h}=$ Altura $(\mathrm{m}) ;$ dap $=$ Diâmetro à $1,30 \mathrm{~cm}$ do solo $(\mathrm{cm}) ; \mathrm{g}=$ Área transversal $\left(\mathrm{cm}^{2}\right) ; \mathrm{v}=$ volume individual $\left(\mathrm{dm}^{3}\right)$. 
TABELA 6 Produção, ICA e IMA para as variáveis analisadas no sítio III.

TABLE 6 Yield, CAI and MAI for the analyzed variables on site III.

\begin{tabular}{|c|c|c|c|c|c|c|c|c|c|c|c|c|}
\hline Idade & $\mathrm{h}$ & ICA h & IMA h & dap & ICA dap & IMA dap & g & ICA g & IMA g & v & ICA v & IMA v \\
\hline 1 & 1,58 & 1,58 & 1,58 & 0,60 & 0,60 & 0,60 & 0,73 & 0,73 & 0,73 & 0,11 & 0,11 & 0,11 \\
\hline 2 & 3,43 & 1,85 & 1,72 & 1,82 & 1,21 & 0,91 & 4,07 & 3,34 & 2,03 & 1,01 & 0,90 & 0,50 \\
\hline 3 & 5,16 & 1,73 & 1,72 & 3,23 & 1,41 & 1,08 & 10,31 & 6,24 & 3,44 & 3,38 & 2,38 & 1,13 \\
\hline 4 & 6,69 & 1,53 & 1,67 & 4,65 & 1,42 & 1,16 & 18,96 & 8,65 & 4,74 & 7,50 & 4,11 & 1,87 \\
\hline 5 & 8,01 & 1,32 & 1,60 & 5,97 & 1,33 & 1,19 & 29,31 & 10,35 & 5,86 & 13,25 & 5,75 & 2,65 \\
\hline 6 & 9,13 & 1,12 & 1,52 & 7,16 & 1,19 & 1,19 & 40,67 & 11,37 & 6,78 & 20,34 & 7,09 & 3,39 \\
\hline 7 & 10,07 & 0,94 & 1,44 & 8,21 & 1,04 & 1,17 & 52,47 & 11,80 & 7,50 & 28,39 & 8,05 & 4,06 \\
\hline 8 & 10,86 & 0,79 & 1,36 & 9,10 & 0,89 & 1,14 & 64,25 & 11,78 & 8,03 & 37,01 & 8,62 & 4,63 \\
\hline 9 & 11,52 & 0,66 & 1,28 & 9,86 & 0,76 & 1,10 & 75,67 & 11,42 & 8,41 & 45,86 & 8,85 & 5,10 \\
\hline 10 & 12,07 & 0,55 & 1,21 & 10,50 & 0,64 & 1,05 & 86,51 & 10,84 & 8,65 & 54,67 & 8,81 & 5,47 \\
\hline 11 & 12,52 & 0,45 & 1,14 & 11,03 & 0,53 & 1,00 & 96,62 & 10,11 & 8,78 & 63,21 & 8,54 & 5,75 \\
\hline 12 & 12,90 & 0,38 & 1,07 & 11,47 & 0,44 & 0,96 & 105,94 & 9,31 & 8,83 & 71,34 & 8,13 & 5,95 \\
\hline 13 & 13,21 & 0,31 & 1,02 & 11,84 & 0,37 & 0,91 & 114,42 & 8,49 & 8,80 & 78,95 & 7,61 & 6,07 \\
\hline 14 & 13,46 & 0,26 & 0,96 & 12,14 & 0,30 & 0,87 & 122,09 & 7,67 & 8,72 & 86,00 & 7,04 & 6,14 \\
\hline 15 & 13,68 & 0,21 & 0,91 & 12,39 & 0,25 & 0,83 & 128,96 & 6,88 & 8,60 & 92,44 & 6,44 & 6,16 \\
\hline 16 & 13,85 & 0,17 & 0,87 & 12,59 & 0,20 & 0,79 & 135,10 & 6,13 & 8,44 & 98,28 & 5,84 & 6,14 \\
\hline 17 & 13,99 & 0,14 & 0,82 & 12,76 & 0,17 & 0,75 & 140,54 & 5,44 & 8,27 & 103,54 & 5,26 & 6,09 \\
\hline 18 & 14,11 & 0,12 & 0,78 & 12,89 & 0,14 & 0,72 & 145,34 & 4,81 & 8,07 & 108,26 & 4,71 & 6,01 \\
\hline 19 & 14,21 & 0,10 & 0,75 & 13,00 & 0,11 & 0,68 & 149,58 & 4,23 & 7,87 & 112,45 & 4,20 & 5,92 \\
\hline 20 & 14,29 & 0,08 & 0,71 & 13,10 & 0,09 & 0,65 & 153,30 & 3,72 & 7,66 & 116,17 & 3,72 & 5,81 \\
\hline 21 & 14,35 & 0,07 & 0,68 & 13,17 & 0,07 & 0,63 & 156,55 & 3,26 & 7,45 & 119,46 & 3,29 & 5,69 \\
\hline 22 & 14,41 & 0,05 & 0,65 & 13,23 & 0,06 & 0,60 & 159,40 & 2,85 & 7,25 & 122,35 & 2,90 & 5,56 \\
\hline 23 & 14,45 & 0,04 & 0,63 & 13,28 & 0,05 & 0,58 & 161,89 & 2,48 & 7,04 & 124,90 & 2,54 & 5,43 \\
\hline
\end{tabular}

Sendo: $\mathrm{h}=$ Altura $(\mathrm{m}) ;$ dap = Diâmetro à $1,30 \mathrm{~cm}$ do solo $(\mathrm{cm}) ; \mathrm{g}=$ Área transversal $\left(\mathrm{cm}^{2}\right) ; \mathrm{v}=$ volume individual $\left(\mathrm{dm}^{3}\right)$.

Nos bracatingais, com $\circ$ passar dos anos, a variável diâmetro é mais fortemente influenciada pela densidade nos sítios de pior qualidade, ocorrendo uma estabilização do crescimento antecipada quando comparada aos demais. A análise das tabelas de incremento corrente dessa variável comprovou essa hipótese, uma vez que, a partir da idade de oito anos, a taxa de incremento do sítio III já era inferior a I,00 $\mathrm{cm}$, enquanto que os demais sítios atingiram esse valor apenas na idade de 10 anos.

Vale destacar que os bracatingais situados em sítios piores possuem densidade inicial elevada em comparação aos melhores sítios (MACHADO et al., 2002), bem como, a mortalidade é mais acentuada até idades próximas aos nove anos, ocorrendo estabilização do número de indivíduos independente do sítio (SOUZA, 20I I). Esse autor acrescentou, ainda que, nos sítios de menor produtividade o autodesbaste é insuficiente para a manutenção do crescimento em diâmetro dos indivíduos nas idades avançadas, fato que não ocorre nos sítios de melhor produtividade por apresentarem densidades iniciais menores.
Machado et al. (200I) identificaram que o sítio exerceu efeito significativo sobre a área transversal. Resultado que também foi observado no presente trabalho, uma vez que a produção foi superior para o sítio I, seguida do sítio II e III. Ocorreu antecipação da culminação do ICA para o sítio III em relação ao sítio II, coincidindo com o sítio I, aos sete anos. Da mesma forma, a intersecção das curvas de ICA e IMA ocorreu aos 13 anos para os sítios I e III e, aos I 5 anos para o sítio II.

A variável área transversal é obtida a partir do diâmetro e também está sujeita a influência da densidade, no entanto, de forma mais expressiva, uma vez que o diâmetro aparece no cálculo de forma potencializada. Característica que foi responsável pela intersecção das curvas de ICA e IMA antecipada no sítio III.

O volume foi a última variável dendrométrica a atingir assíntota de crescimento. Para o sítio I, o valor assintótico de produção $\left(b_{0}\right)$ foi de $548,52 \mathrm{dm}^{3}$, superior à produção do sítio II $\left(5|5,4| \mathrm{dm}^{3}\right)$, seguida do sítio III $\left(142,03 \mathrm{dm}^{3}\right)$. Esse resultado reforça a conclusão encontrada por Machado et al. (200I), os quais relataram efeito positivo do sítio sobre essa variável paraa Bracatinga. 
O máximo ICA foi observado aos nove anos para os sítios I e III; para o sítio II ocorreu na idade de 13 anos. A intersecção das curvas de ICA e IMA ocorreu, antecipadamente, para o sítio III, aos 16 anos, enquanto que no sítio I ocorreu aos 17 anos e no sítio II aos 23 anos.

Hess e Schneider (2010) concluíram, por meio do estudo do crescimento em volume da Araucaria angustifolia em diferentes sítios no estado do Rio Grande do Sul que a produtividade e a idade de crescimento máximo destas foram diferentes em cada sítio. Esse fato deve-se aos aspectos físicos e condições bióticas da região, como: altitude, conteúdo nutricional do solo, precipitação, etc.

\section{CONCLUSÕES}

Todos os modelos ajustados apresentaram distribuição de resíduos homogênea, independente e pouco dispersa ao longo da linha zero, no entanto, Chapman-Richards foi selecionado pelo melhor desempenho geral definido pelo ranking.

As intersecções das curvas de ICA e IMA seguiram a ordem cronológica esperada, primeiramente para altura, seguida pelo diâmetro, área transversal e volume em todas as classes de sítio.

O sítio afetou, de forma positiva, a evolução das variáveis dendrométricas estudadas, exercendo maior influência sobre a altura como era esperado.

\section{REFERÊNCIAS}

ASSMANN, E. The principles of forest yield study. Oxford: Pergamon, 1970. $506 \mathrm{p}$.

BARUSSO, A. P. Determinação de funções de crescimento mediante análise de tronco. 1977. 133 f. Dissertação (Mestrado em Engenharia Florestal) - Universidade Federal do Paraná, Curitiba, 1977.

CARPANEZZI, A. A.; LAURENT, J. E.; BAGGIO, A. J. Manual técnico da Bracatinga (Mimosa scabrella Bentham). Curitiba: EMBRAPA-CNPF, 1988. 70 p. (Série Documentos, 20).

FERRAZ FILHO, A. C.; SCOLFORO, J. R. S.; FERREIRA, M. Z.; MAESTRI, R.; ASSIS, A. L.; OLIVEIRA, A. D.; MELLO, J. M. Dominant height projection model with the addition of environmental variables. Cerne, Lavras, v. 17, n. 3, p. 427-433, 2011.

FINGER, C. A. G. Fundamentos da biometria florestal. Santa Maria: UFSM/CEPEF/FATEC, 1992. 269 p.
HESS, A. F; SCHNEIDER, P. R. Crescimento em volume de Araucaria angustifolia (Bertol.) Kuntze em três regiões do Rio Grande do Sul, Brasil. Ciência Florestal, Santa Maria, v. 20, n. I, p. 107-122, 2010.

MACHADO, S. A.; SILVA, L. C. R.; FIGURA, M. A.; TÉO, S. J.; NASCIMENTO, R. G. M. Comparison of methods for estimating heights from complete stem analysis data for Pinus taeda. Ciência Florestal, Santa Maria, v. 20, n. I, p. 45-55, 2010.

MACHADO, S. A.; SOUZA, R. F;; JASKIU, E.; CAVALHEIRO, $R$. Construction of site curves for native Mimosa scabrella stands in the metropolitan region of Curitiba. Cerne, Lavras, v. 17, n. 4, p. 489-497, 201 I.

MACHADO,S.A.; TONON, A. E.; OLIVEIRA, E. B.; FIGUEIREDO FILHO, A.; CARPANEZZI, A. A. Efeitos da densidade inicial e do sítio sobre o desenvolvimento de bracatingais nativos da região metropolitana de Curitiba. Boletim de Pesquisa Florestal, Colombo, n. 43, p. 19-46, 2001.

MACHADO, S. A.; TONON, A. E. N.; FIGUEIREDO FILHO, A.; OLIVEIRA, E. B. Comportamento da mortalidade natural em bracatingais nativos em diferentes densidades iniciais e classes de sítio. Ciência Florestal, Santa Maria, v. I2, n. 2, p. 4I-50, 2002.

NUTTO, L. Manejo do crescimento diamétrico de Araucaria angustifolia (Bert.) O. Ktze. baseado na árvore individual. Ciência Florestal, Santa Maria, v. II, n. 2, p. 9-25, 200 I.

SANQUETTA, C. R.; WEBER, S. H.; FORMAGINI, F; BARBEIRO, L. S.; VIEIRA, G. Equações individuais e determinação de classes de crescimento para Nectandra grandiflora Ness. a partir de dados de análise de tronco. Scientia Agrária, Piracicaba, v. II, n. I, p. I-8, 2010.

SCOLFORO, J. R. S.; MACHADO, S. A. Curvas de índice de sítio para plantações de Pinus taeda nos estados do Paraná e Santa Catarina. Floresta, Curitiba, v. 18, n. I/2, p. I59173, 1988.

SILVA, V. P. da; MAZUCHOWSKI, J. Z.; PINTO, A. F; MINIOLLI NETTO, J.; BECKER, J. C. Memórias da oficina sobre bracatinga no Vale do Ribeira: 2004, Curitiba, PR. Colombo: EMBRAPA Florestas, 2006. 107 p.

SOUZA, R. F. Modelagem da biomassa aérea e do carbono do povoamento de Mimosa Scabrella Benth. em bracatingais nativos da região metropolitana de Curitiba. 201 I. 106 f. Dissertação (Mestrado em Engenharia Florestal) - Universidade Federal do Paraná, Curitiba, 201 I.

TONINI, H.; COSTA, M. C. G.; SCHWENGBER, L. A. M. Crescimento da teca (Tectona grandis) em reflorestamento na Amazônia Setentrional. Pesquisa Florestal Brasileira, Colombo, n. 59, p. 5-14, 2009. 
TONINI, H.; FINGER, C. A. G.; SCHNEIDER, P. R.; SPATHELF,

P. Crescimento em altura de Pinus Elliottii Engelm., na região de Piratini no Rio Grande do Sul, Brasil. Ciência Rural, Santa Maria, v. 3I, n. 3, p. 4I7-423, 2001.
TONINI, H.; OLIVEIRAJUNIOR, M. M. C.; SCHWENGBER, D. Crescimento de espécies nativas da Amazônia submetidas ao plantio no estado de Roraima. Ciência Florestal, Santa Maria, v. 18, n. 2, p. 15I-158, 2008. 
